\title{
Implementation without expected utility: ex-post verifiability
}

\section{Hitoshi Matsushima ${ }^{1}$}

Received: 4 September 2018 / Accepted: 12 June 2019 / Published online: 18 June 2019 (c) The Author(s) 2019

\begin{abstract}
This study investigates implementation of a social choice function with complete information, where we impose various restrictions such as boundedness, permission of only small transfers, and uniqueness of iterative dominance in strict terms. We assume that the state is ex-post verifiable after the determination of allocation. We show that with three or more players, any social choice function is uniquely and exactly implementable in iterative dominance. Importantly, this study does not assume either expected utility or quasi-linearity, even if we utilize the stochastic method of mechanism design explored by Abreu and Matsushima (Econometrica 60:993-1008, 1992a; Econometrica 60:1439-1442, 1992b; J Econ Theory 64(1):1$19,1994)$. We further show that even with incomplete information, and even with two players, any ex-post incentive compatible social choice function is uniquely and exactly implementable in iterative dominance.
\end{abstract}

\section{Introduction}

This study investigates unique and exact implementation of a social choice function under complete information. We assume that the state is ex-post verifiable. We design stochastic mechanisms but do not assume either expected utility or quasi-linearity.

The central planner attempts to achieve the allocation implied by the social choice function that is contingent on the state. The central planner, however, cannot observe the state before determining an allocation. Hence, he (or she) designs a mechanism to induce informed players to reveal their knowledges about the state. This mechanism must incentivize them to make the desirable (i.e., truthful) announcements as

Hitoshi Matsushima

hitoshi@e.u-tokyo.ac.jp

1 Department of Economics, University of Tokyo, Hongo, Bunkyo-ku, Tokyo 113-0033, Japan 
unique equilibrium behavior. The problem of this study is to clarify whether the central planner can design such effective mechanisms. ${ }^{1}$

We assume that the state becomes verifiable after the central planner determines the allocation. For instance, by conducting a follow-up survey, the central planner obtains a resultant verifiable consequence of the allocation decision that includes information about the state. The central planner utilizes this information for expost monetary transfers with players as a clue to detecting their lying. We show that by making ex-post monetary transfers contingent on this verified information as well as their announcements, the central planner can design a mechanism to effectively penalize any detected liar, making all players willing to make their desirable announcements.

Owing to this ex-post verifiability, we can impose the following various severe restrictions on mechanism design. We use iterative dominance in strict terms as the solution concept, which is defined as the set of all strategy profiles that survive through the iterative removal of strictly dominated strategies. We impose the uniqueness of such an iteratively undominated strategy for each player, even if iterative dominance is a very weak solution concept. Next, we require a mechanism to be bounded in the terminology of Jackson (1992); we consider only mechanisms whose message spaces are finite. Moreover, we use only small monetary transfers; we require any transfer to be close to zero off the equilibrium path, and even no transfers on the equilibrium path.

We show that any social choice function is uniquely, and exactly, implementable in iterative dominance, where we design a bounded mechanism, use only small transfers, and make no transfers on the equilibrium path. Importantly, we do not assume either expected utility or quasi-linearity, even if we design a stochastic mechanism according to the method explored by Abreu and Matsushima (1992a, 1994). All we need on utility functions in this study is a much weaker condition than expected utility and quasi-linearity; each player's utility function is continuous in monetary transfer and lottery, and is increasing in monetary transfer.

It is generally important to investigate various aspects of bounded rationality in the implementation literature. This study designs a mechanism that has the unique equilibrium at every state; this equilibrium implies a simple honest-reporting behavior. However, to make players recognize that this is the unique one, the designed mechanism may force them to take an involved rational thinking. The number of iterations of reasoning to reach the uniqueness is an example of such complexity measures in iterative dominance. Glazer and Rosenthal (1992) pointed out that there is a trade-off between the length of this iteration and a focal point. Arya et al. (1995) demonstrated a simple mechanism in which two rounds of iterations are enough to reach the uniqueness. As a response to Glazer and Rosenthal, Abreu and Matsushima (1992b) emphasized the importance of public recognition that complicated iterations can be reduced to simple iterations of a single step argument.

\footnotetext{
1 For surveys on implementation theory, see Moore (1992), Palfrey (1992), Osborne and Rubinstein (1994, Chapter 10), Jackson (2001), and Maskin and Sjöström (2002).
} 
In contrast to these works, this study focuses on another question on bounded rationality; can a player properly make the desirable choice at a step even if he (or she) is not the expected-utility maximizer? This study gives a positive answer to this question.

It is well known in the implementation literature that with no ex-post verifiability, Makin-monotonicity is a necessary condition for a social choice function to be implementable in Nash equilibrium (e.g., Maskin 1999). Maskin-monotonicity is a quite demanding condition for a deterministic social choice function. Matsushima (1988) and Abreu and Sen (1991) demonstrated a device of virtualness, which can drastically calm this difficulty by approximating a deterministic social choice function to a stochastic social choice function. However, this virtualness crucially depends on expected utility.

In contrast, this study assumes ex-post verifiability. With ex-post verifiability, Maskin-monotonicity is no longer necessary. Because of this irrelevance, we can apply the Abreu-Matsushima stochastic method even to the case without expected utility (also without quasi-linearity) with ease. The functioning of the stochastic method a la Abreu and Matsushima relies just on the local linearity of preferences, which is automatically implied by the above-mentioned continuity and increasingness.

There exist previous works such as Hansen (1985), Mezzetti (2004), and Deb and Mishra (2014) that incorporated verifiability into mechanism design. These works showed that verifiability makes incentive compatibility more easily satisfied. In contrast, this study's concern is the impact of verifiability on uniqueness. In this respect, this study is related to Kartik and Tercieux (2012) and Ben-Porath and Lipmann (2012), which investigated full implementation with hard evidence, stating that the great degree to which each player's showing hard evidences directly proves his (or her) announcement to be correct is crucial in full implementation. The companion paper (e.g., Matsushima 2017) extends this study's result to the case in which the state is only partially verifiable.

The organization of this study is as follows. Section 2 shows the model that assumes continuity and increasingness, instead of expected utility and quasi-linearity. Section 3 explains small monetary transfers and ex-post verifiability. Section 4 introduces iterative dominance. Section 5 constructs a mechanism that is finite and stochastic. Section 6 shows the main theorem.

Section 7 generalizes this study's results to incomplete information with two or more players. We show that even with incomplete information and even with two players, any ex-post incentive compatible social choice function is uniquely, and exactly, implementable in iterative dominance in the same manner as complete information with three or more players. Section 8 concludes.

\section{The model}

We consider a situation in which the central planner determines an allocation and makes small monetary transfers. Let $N \equiv\{1, \ldots, n\}$ denote the finite set of all players, where $n \geq 3$. Let $A$ denote the finite set of all allocations. Let $\Delta$ denote 
the set of all lotteries over allocations. We denote $\alpha \in \Delta$. We will write $\alpha=a$ if $\alpha(a)=1$. Let $\Omega$ denote the finite set of all states. A social choice function is defined as $f: \Omega \rightarrow \Delta$. For every $\omega \in \Omega$, the lottery over allocations $f(\omega) \in \Delta$ implies the most desirable one to achieve at the state $\omega$. This study considers both deterministic and stochastic social choice functions.

We define the state-contingent utility function for each player $i \in N$ as

$$
u_{i}: \Delta \times R \times \Omega \rightarrow R,
$$

where $u_{i}\left(\alpha, t_{i}, \omega\right)$ implies player $i^{\prime} s$ utility when he (or she) expects the state $\omega$ to occur, expects the central planner to determine the allocation according to the lottery $\alpha \in \Delta$ and make a monetary transfer $t_{i} \in R$ to him.

We assume that $u_{i}\left(\alpha, t_{i}, \omega\right)$ is continuous with respect to $\alpha \in \Delta$ and $t_{i} \in R$, and that $u_{i}\left(\alpha, t_{i}, \omega\right)$ is increasing in $t_{i}$. This study does not assume either expected utility or quasi-linearity.

\section{Small transfers and ex-post verifiability}

Fix an arbitrary positive real number $\varepsilon>0$. Because of players' limited liability, the central planner cannot make any monetary transfer that is greater than $\varepsilon$. We can fix $\varepsilon$ as close to zero as possible.

The state is common knowledge among players from the beginning. However, the central planner can observe the state only after he (or she) determines the allocation. Hence, the state becomes verifiable only in the ex-post term. The central planner can make ex-post monetary transfers contingent on the state as well as the players' messages. The central planner cannot make his allocation selection contingent on the state because he must determine the allocation before he observes the state.

Based on these observations, we define a mechanism as $\mathrm{G} \equiv(M, g, x)$, where $M \equiv \underset{i \in N}{\times} M_{i}, M_{i}$ denotes the set of all messages of player $i, g: M \rightarrow \Delta$ denotes the allocation rule, $x \equiv\left(x_{i}\right)_{i \in N}$ denotes the transfer rule, and $x_{i}: M \times \Omega \rightarrow[0, \varepsilon]$ denotes the transfer rule for player $i$. We assume that $M_{i}$ is finite for all $i \in N$; we focus on mechanisms that are bounded (Jackson 1992). ${ }^{2}$

Each player $i \in N$ announces a message $m_{i} \in M_{i}$ to the central planner, which is contingent on the state $\omega$. The central planner determines the allocation according to the lottery $g(m) \in \Delta$ implied by the message profile $m \equiv\left(m_{i}\right)_{i \in N} \in M$ and the allocation rule $g$. After the state $\omega$ becomes verifiable, the central planner receives the monetary transfer $x_{i}(m, \omega) \in[0, \varepsilon]$ from each player $i$, which is implied by the message profile $m \in M$, the state $\omega \in \Omega$, and the transfer rule $x_{i}$ for player $i$.

A strategy for each player $i$ is defined as $s_{i}: \Omega \rightarrow M_{i}$, according to which player $i$ announces the message $s_{i}(\omega) \in M_{i}$ when he observes $\omega \in \Omega$. Let $S_{i}$ denote the set of all strategies for player $i$. Let $S \equiv \underset{i \in N}{\times} S_{i}$ and $s \equiv\left(s_{i}\right)_{i \in N} \in S$.

\footnotetext{
${ }^{2}$ Without substantial changes, we can eliminate the assumption of finiteness on the state space, where we need to construct mechanisms that does not satisfy finiteness but does satisfy the boundedness in the original definition in Jackson (1992).
} 


\section{Iterative dominance}

We introduce a solution concept namely iterative dominance, which is defined as the survival of iterative removal of messages that are strictly dominated. For every $i \in N$ and $\omega \in \Omega$, let

$$
M_{i}(0, \omega) \equiv M_{i} \text {. }
$$

Recursively, for each $h \geq 1$, we define a subset of player $i^{\prime} s$ messages $M_{i}(h, \omega) \subset M_{i}$ in the manner that $m_{i} \in M_{i}(h, \omega)$ if and only if $m_{i} \in M_{i}(h-1, \omega)$, and there exists no $m_{i}^{\prime} \in M_{i}(h-1, \omega)$ such that for every $m_{-i} \in M_{-i}(h-1, \omega)$,

$$
u_{i}\left(g\left(m_{i}^{\prime}, m_{-i}\right),-x_{i}\left(m_{i}^{\prime}, m_{-i}, \omega\right), \omega\right)>u_{i}\left(g(m),-x_{i}(m, \omega), \omega\right),
$$

where we denote $M_{-i}(h-1, \omega) \equiv \underset{j \in N \backslash\{i\}}{\times} M_{j}(h-1, \omega)$. In this definition, in order to eliminate a message $m_{i} \in M_{i}(h-1, \omega)$, we require player $i$ to strictly prefer another message $m_{i}^{\prime}$ to $m_{i}$ irrespective of the other players' messages $m_{-i} \in M_{-i}(h-1, \omega)$. Let

$$
M_{i}(\infty, \omega) \equiv \bigcap_{h=0}^{\infty} M_{i}(h, \omega) .
$$

Definition 1 Astrategy $s_{i} \in S_{i}$ for player $i$ is said to be iteratively undominated in a mechanism $G$ if

$$
s_{i}(\omega) \in M_{i}(\infty, \omega) \text { for all } \omega \in \Omega .
$$

Definition 2 A mechanism $G$ is said to uniquely implement a social choice function $f$ in iterative dominance if there exists the unique iteratively undominated strategy profile $s=\left(s_{i}\right)_{i \in N} \in S$ in $G$, i.e.,

$$
M_{i}(\infty, \omega)=\left\{s_{i}(\omega)\right\} \quad \text { for all } \omega \in \Omega \text { and } i \in N,
$$

and it induces the value of the SCF and no monetary transfers at all times, i.e., for every $\omega \in \Omega$,

$$
g(s(\omega))=f(\omega)
$$

and

$$
x_{i}(s(\omega))=0 \text { for all } i \in N .
$$

A social choice function $f$ is said to be uniquely implementable in iterative dominance if there exists a bounded (finite) mechanism $G$ that uniquely implements $f$ in iterative dominance.

Iterative dominance is a very weak solution concept. Unique implementation in iterative dominance automatically implies unique implementation in mixed strategy Nash equilibrium. This unique implementation requires not only the achievement of allocations implied by the social choice function but also zero monetary 
transfers on the equilibrium path. Even off the equilibrium path, only small transfers are permitted.

\section{Construction}

Fix an arbitrary real numbers $\eta_{1} \in(0, \varepsilon)$. Let $\eta_{2} \equiv \varepsilon-\eta_{1}>0$. Fix an arbitrary integer $K>0$. Fix an arbitrary allocation $a^{*} \in A$. By using the stochastic method explored by Abreu and Matsushima (1992a, 1994), we construct a mechanism, which is denoted by $G^{*}=G^{*}\left(f, \eta_{1}, \eta_{2}, K, a^{*}\right)=(M, g, x)$, in the following manner. For every $i \in N$, let

$$
M_{i}=\underset{k=1}{\stackrel{K}{\times}} M_{i}^{k}
$$

and

$$
M_{i}^{k}=\Omega \quad \text { for all } k \in\{1, \ldots, K\} .
$$

Each player $i \in N$ announces $K$ multiple sub-messages about the state, i.e., $m_{i}^{k} \in \Omega$ for all $k \in\{1, \ldots, K\}$, at once, where we denote $m_{i}=\left(m_{i}^{k}\right)_{k=1}^{K}$. Let $M^{k} \equiv \underset{i \in N}{\times} M_{i}^{k}$ and $m^{k}=\left(m_{i}^{k}\right)_{i \in N} \in M^{k}$.

For each $k \in\{1, \ldots, K\}$, we define $g^{k}: M^{k} \rightarrow \Delta$ in the manner that for every $\omega \in \Omega$,

$$
g^{k}\left(m^{k}\right)=f(\omega) \quad \text { if } \quad m_{i}^{k}=\omega \quad \text { for at least } n-1 \text { players, }
$$

and

$$
g^{k}\left(m^{k}\right)=a^{*} \quad \text { if there exists no such } \omega .
$$

Note that $g^{k}$ is well-defined because of $n \geq 3$. Let

$$
g(m)=\frac{\sum_{k=1}^{K} g^{k}\left(m^{k}\right)}{K} \text { for all } \quad m \in M .
$$

The interpretation of the specified allocation rule $g$ in $G^{*}$ is as follows. The central planner randomly selects an integer $k$ from $\{1, \ldots, K\}$ with the same probability and determines an allocation according to the corresponding lottery $g^{k}\left(m^{k}\right) \in \Delta$. In this case, the central planner can select an allocation according to the lottery $f(\omega)$ implied by the social choice function $f$ and the state $\omega$ whenever at least $n-1$ players $i$ announce $m_{i}^{k}=\omega$. If there exists no such $\omega$, he selects $a^{*}$. Since the central planner selects $k$ at random, he selects each allocation $a \in A$ with the probability given by

$$
g(m)(a)=\frac{\sum_{k=1}^{K} g^{k}\left(m^{k}\right)(a)}{K} .
$$

We specify the transfer rule $x_{i}$ for each player $i$ as follows: for every $(m, \omega) \in M \times \Omega$, 


$$
\begin{aligned}
x_{i}(m, \omega)=\eta_{1}+\frac{r_{i}\left(m_{i}\right)}{K} \eta_{2} \quad & \text { if there exists } k \in\{1, \ldots, K\} \text { such that } \\
& m_{i}^{k} \neq \omega, \text { and } \\
& m_{j}^{k^{\prime}}=\omega \text { for all } k^{\prime}<k \text { and } j \in N,
\end{aligned}
$$

and

$$
x_{i}(m, \omega)=\frac{r_{i}\left(m_{i}\right)}{K} \eta_{2} \quad \text { if there exists no such } k \in\{1, \ldots, K\},
$$

where $r_{i}\left(m_{i}\right) \in\{0, \ldots, K\}$ denotes the number of the integers $k \in\{1, \ldots, K\}$ such that $m_{i}^{k} \neq \omega$.

The interpretation of the specified transfer rule $x_{i}$ for player $i$ in $G^{*}$ is as follows. If a player $i$ is one of the first deviants from $\omega$, i.e., one of the players who tell lies as the earliest sub-message among all deviants, he is fined the monetary amount $\eta_{1}$.

Player $i$ is also fined the monetary amount $\frac{r_{i}\left(m_{i}\right)}{K} \eta_{2}$ in proportion to the number of his dishonest sub-messages. (Note that announcing all his sub-messages dishonestly, he is fined the monetary amount $\eta_{1}+\eta_{2}$ in totality.)

Note from the specifications of $\left(\eta_{1}, \eta_{2}\right)$ that for every $i \in N$ and $(m, \omega) \in M \times \Omega$,

$$
0 \leq x_{i}(m, \omega) \leq \eta_{1}+\eta_{2}=\varepsilon .
$$

Hence, the central planner never makes monetary transfers that are greater than $\varepsilon$.

We denote $s_{i}=\left(s_{i}^{k}\right)_{k=1}^{K}$, where $s_{i}^{k}: \Omega \rightarrow M_{i}^{k}$. We define the honest strategy for player $i, s_{i}^{*}=\left(s_{i}^{* k}\right)_{k=1}^{K}$, in $G^{*}$ as

$$
s_{i}^{* k}(\omega)=\omega \text { for all } k \in\{1, \ldots, K\} \quad \text { and } \omega \in \Omega .
$$

According to $s_{i}^{*}$, player $i$ makes the honest announcement for every sub-message. The honest strategy profile $s^{*} \equiv\left(s_{i}^{*}\right)_{i \in N}$ induces the value of the SCF $f$, i.e.,

$$
g\left(s^{*}(\omega)\right)=f(\omega) \text { for all } \omega \in \Omega,
$$

and induces no monetary transfers, i.e.,

$$
x_{i}\left(s^{*}(\omega), \omega\right)=0 \quad \text { for all } i \in N \text { and } \omega \in \Omega .
$$

Since $u_{i}\left(\alpha, t_{i}, \omega\right)$ is continuous in $\left(\alpha, t_{i}\right)$ and increasing in $t_{i}$. we can specify $K$ sufficiently large in the manner that whenever

1. $\max _{a \in A}\left|\alpha(a)-\alpha^{\prime}(a)\right| \leq \frac{1}{K}$

then

2. $u_{i}\left(\alpha,-t_{i}, \omega\right)>u_{i}\left(\alpha^{\prime},-t_{i}-\eta_{1}, \omega\right)$ for all $t_{i} \in\left[0, \eta_{2}\right]$ and $\omega \in \Omega$.

The inequalities (2) imply that the loss from the monetary fine $\eta_{1}$ is greater than the gain from any change of stochastic allocation within the limit implied by (1). 


\section{The theorem}

The following theorem indicates that the above-specified mechanism $G^{*}$ uniquely implements the social choice function $f$ in iterative dominance. Since $G^{*}$ is welldefined, we can conclude that with ex-post verifiability and with more than two players, any social choice function is uniquely implementable in iterative dominance even if players' utility functions do not satisfy either expected utility or quasi-linearity, where we need no monetary transfers on the equilibrium path and almost no monetary transfers even off the equilibrium path.

Theorem The honest strategy profile $s^{*}$ is the unique iteratively undominated strategy profile in $G^{*}$.

Proof We can show that each player $i \in N$ prefers $m_{i}^{1}=\omega$ as follows. Suppose that there exists another player $j \in N \backslash\{i\}$ who announces $m_{j}^{1} \neq \omega$. In this case, by announcing $m_{i}^{1} \neq \omega$ instead of $\omega$, player $i$ is fined $\eta_{1}$ or even more, while the resultant change of allocation is within the limit implied by (1). Hence, from (2), the impact of the fine $\eta_{1}$ on his welfare is greater than the impact of the resultant change of allocation.

Next, suppose that there exists no player $j \in N \backslash\{i\}$ other than player $i$ who announces $m_{j}^{1} \neq \omega$. Then, by announcing $m_{i}^{1} \neq \omega$ instead of $\omega$, player $i$ is fined $\frac{\eta_{2}}{K}$ or even more. (Note that even if he announces $m_{i}^{1}=\omega$, he may be one of the first deviants, and therefore, he does not necessarily avoid the fine $\eta_{1}$.) From the specification of $g$ and $n \geq 3$, there is no resultant change of allocation. From these observations, he prefers $m_{i}^{1}=\omega$ regardless of the other players' announcements.

Fix an arbitrary integer $h \in\{2, \ldots, K\}$. Suppose that any player $i \in N$ announces $m_{i}^{h^{\prime}}=\omega$ for all $h^{\prime} \in\{1, \ldots, h-1\}$. Suppose that there exists a player $j \in N \backslash\{i\}$ other than player $i$ who announces $m_{j}^{h} \neq \omega$. Then, by announcing $m_{i}^{h} \neq \omega$ instead of $\omega$, player $i$ is fined $\eta_{1}$ or even more. In the same manner as above, the impact of the fine $\eta_{1}$ on his welfare is greater than the impact of the resultant change of allocation. Next, suppose that there exists no player $j \in N \backslash\{i\}$ other than player $i$ who announces $m_{j}^{h} \neq \omega$. Then, by announcing $m_{i}^{h} \neq \omega$ instead of $\omega$, player $i$ is fined $\frac{\eta_{2}}{K}$ or even more. In the same manner as above, there is no resultant change of allocation in this case. Hence, he prefers $m_{i}^{h}=\omega$

The proof of the theorem is similar to the proofs of the main theorems in Abreu and Matsushima (1992a, 1994). However, there is a substantial difference between these works and this study; Abreu and Matsushima did not use ex-post verifiability. It is well known in the implementation literature that without ex-post verifiability, Maskin-monotonicity is a necessary condition for a social choice function to be implementable in Nash equilibrium. Maskin-monotonicity is a quite demanding requirement if we consider deterministic social choice functions.

Matsushima (1988) and Abreu and Sen (1991) showed that a deterministic social choice function fails to satisfy Maskin-monotonicity, but there always exists a stochastic social choice function that is virtually the same as this deterministic social 
choice function and satisfies Maskin-monotonicity. However, this virtualness relies crucially on expected utility; we cannot directly extend Abreu and Matsushima (1992a, 1994) to the case without expected utility.

In contrast, this study assumes ex-post verifiability. With ex-post verifiability, Maskin-monotonicity is no longer a necessary condition. Abreu and Matsushima (1992a, 1994) developed a stochastic method of iteratively eliminating unwanted equilibria. The main theorem of this study shows that this stochastic method functions even without expected utility; all we need to apply this method to the case without expected utility is just to assume that $u_{i}\left(\alpha, t_{i}, \omega\right)$ is continuous in $\left(\alpha, t_{i}\right)$ and increasing in $t_{i}$.

\section{Incomplete information and two players}

We have assumed complete information with which each player commonly receives the full information about the state. This section eliminate this assumption; Each player $i \in N$ observes his (or her) private signal $\omega_{i} \in \Omega_{i}$, where $\Omega_{i}$ is a non-empty and finite set. We define the set of states $\Omega$ as a subset of $\underset{i \in N}{\times} \Omega_{i}$. Each player cannot observe the other players' private signals. Hence, we modify Definition 2 by additionally requiring the unique iteratively undominated strategy $s_{i}$ to be independent of $\omega_{-i} \equiv\left(\omega_{j}\right)_{j \neq i}$.

We introduce ex-post incentive compatibility as follows.

Definition 3 A social choice function $f$ is said to be ex-post incentive compatible if there exists a function $\tilde{f}: \underset{i \in N}{\times} \Omega_{i} \rightarrow \Delta$ such that

$$
\tilde{f}(\omega)=f(\omega) \text { for all } \omega \in \Omega,
$$

and

$$
u_{i}(\tilde{f}(\omega), 0, \omega) \geq u_{i}\left(\tilde{f}\left(\omega_{i}^{\prime}, \omega_{-i}\right), 0, \omega\right) \quad \text { for all } \quad i \in N, \omega \in \Omega \text {, and } \omega_{i}^{\prime} \in \Omega_{i} .
$$

Note that with complete information and with three or more players, any social choice function automatically satisfies ex-post incentive compatibility by setting $\tilde{f}=g^{1}$ (in the proof of the Theorem). Note also that ex-post incentive compatibility is a necessary condition for implementation in iterative dominance.

For the purpose of this section, we modify the mechanism constructed in Sect. 5 (i.e., $G^{*}$ ) by replacing $f$ and $M_{i}^{k}=\Omega$ with $\tilde{f}$ and $M_{i}^{k}=\Omega_{i}$, and by setting $g^{k}=\tilde{f}$. We further modify the honest strategy $s_{i}^{*}$ for each player $i$ as

$$
s_{i}^{* k}(\omega)=\omega_{i} \text { for all } k \in\{1, \ldots, K\} \text { and } \omega \in \Omega .
$$

With these modifications, we can directly apply the proof of the Theorem to the case of incomplete information with two or more players; whenever a social choice function $f$ is ex-post incentive compatible, in the modified mechanism $G^{*}$, the modified honest strategy profile $s^{*}$ is the unique iteratively undominated strategy profile. Hence, even 
with incomplete information and even with two or more players, any ex-post incentive compatible social choice function is uniquely and exactly implementable in iterative dominance, where we do not assume either expected utility or quasi-linearity, no side payments are permitted on the equilibrium path, only small side payments are permitted off the equilibrium path, and the constructed mechanism satisfies boundedness. ${ }^{3}$

\section{Conclusion}

We investigated unique and exact implementation of a social choice function under complete information, where we required a mechanism to be bounded, utilize only small monetary transfers, and satisfy uniqueness of iterative dominance. By assuming that the state is ex-post verifiable, we showed that any social choice function is uniquely and exactly implementable in iterative dominance. This permissive result does not assume either expected utility or quasi-linearity, even if stochastic mechanisms are used. This study is the first work to analyze bounded mechanism design with uniqueness of mixed strategy Nash equilibrium without expected utility.

Acknowledgements The earlier version corresponds to Sect. 4 in Matsushima (2017). However, this study includes substantial arguments that the earlier version did not make. This study was supported by a grantin-aid for scientific research (KAKENHI 25285059) from the Japan Society for the Promotion of Science (JSPS) and the Ministry of Education, Culture, Sports, Science and Technology (MEXT) of the Japanese government. I am grateful to the editor and an anonymous referee for their useful comments. All errors are mine.

Open Access This article is distributed under the terms of the Creative Commons Attribution 4.0 International License (http://creativecommons.org/licenses/by/4.0/), which permits unrestricted use, distribution, and reproduction in any medium, provided you give appropriate credit to the original author(s) and the source, provide a link to the Creative Commons license, and indicate if changes were made.

\section{References}

Abreu D, Matsushima H (1992a) Virtual implementation in iteratively undominated strategies: complete information. Econometrica 60:993-1008

Abreu D, Matsushima H (1992b) A response to glazer and rosenthal. Econometrica 60:1439-1442

Abreu D, Matsushima H (1994) Exact implementation. J Econ Theory 64(1):1-19

Abreu D, Sen A (1991) Virtual implementation in nash equilibrium. Econometrica 59:997-1021

Arya A, Glover J, Young R (1995) Virtual implementation in separable Bayesian environments using simple mechanisms. Games Econ Behav 9:127-138

Ben-Porath E, Lipman B (2012) Implementation with partial provability. J Econ Theory 147:1689-1724

Deb R, Mishra D (2014) Implementation with contingent contracts. Econometrica 82:2371-2393

Glazer J, Rosenthal R (1992) A note on Abreu-Matsushima mechanisms. Econometrica 60:1435-1438

Hansen R (1985) Auctions with contingent payments. Am Econ Rev 75:862-865

Jackson M (1992) Implementation in undominated strategies: a look at bounded mechanisms. Rev Econ Stud 59:757-775

Jackson M (2001) A crash course in implementation theory. Soc Choice Welf 18:655-708

\footnotetext{
${ }^{3}$ With the assumption of expected utility, we can make the same argument as in this section even if we replace ex-post incentive compatibility and iterative dominance with Bayesian incentive compatibility and some modified definition of iterative dominance in the interim term, although details are omitted in this study.
} 
Kartik N, Tercieux O (2012) Implementation with evidence. Theor Econ 7:323-356

Maskin E (1999) Nash equilibrium and welfare optimality. Rev Econ Stud 66:23-38

Maskin E, Sjöström T (2002) Implementation theory. In: Arrow K, Sen A, Suzumura K (eds) Handbook of social choice and welfare, vol 1. Elsevier, Amsterdam

Matsushima H (1988) A new approach to the implementation problem. J Econ Theory 45:128-144

Matsushima H (2017) Dynamic implementation, verification, and detection. University of Tokyo, Tokyo (CARF-F-416)

Mezzetti C (2004) Mechanism design with interdependent valuations: efficiency. Econometrica $72: 1617-1626$

Moore J (1992) Implementation in environments with complete information. In: Laffont JJ (ed) Advances in economic theory: sixth world congress. Cambridge University Press, Cambridge

Osborne M, Rubinstein A (1994) A course in game theory. MIT Press, Cambridge

Palfrey T (1992) Implementation in Bayesian equilibrium: the multiple equilibrium problem in mechanism design. In: Laffont JJ (ed) Advances in economic theory: sixth world congress. Cambridge University Press, Cambridge

Publisher's Note Springer Nature remains neutral with regard to jurisdictional claims in published maps and institutional affiliations. 